\title{
Fluxon propagation and Fiske steps in long Josephson tunnel junctions
}

\author{
Erné, S. N.; Ferrigno, A.; Parmentier, R. D.
}

Published in:

Physical Review B

Link to article, DOI:

10.1103/PhysRevB.27.5440

Publication date:

1983

Document Version

Publisher's PDF, also known as Version of record

Link back to DTU Orbit

Citation (APA):

Erné, S. N., Ferrigno, A., \& Parmentier, R. D. (1983). Fluxon propagation and Fiske steps in long Josephson tunnel junctions. Physical Review B, 27(9), 5440-5446. https://doi.org/10.1103/PhysRevB.27.5440

\section{General rights}

Copyright and moral rights for the publications made accessible in the public portal are retained by the authors and/or other copyright owners and it is a condition of accessing publications that users recognise and abide by the legal requirements associated with these rights.

- Users may download and print one copy of any publication from the public portal for the purpose of private study or research.

- You may not further distribute the material or use it for any profit-making activity or commercial gain

- You may freely distribute the URL identifying the publication in the public portal

If you believe that this document breaches copyright please contact us providing details, and we will remove access to the work immediately and investigate your claim 


\title{
Fluxon propagation and Fiske steps in long Josephson tunnel junctions
}

\author{
S. N. Erné and A. Ferrigno \\ Physikalisch-Technische Bundesanstalt, Institut Berlin, D-1000 Berlin 10, West Germany \\ R. D. Parmentier* \\ Laboratory of Applied Mathematical Physics, The Technical University of Denmark, \\ DK-2800 Lyngby, Denmark \\ (Received 15 September 1982)
}

\begin{abstract}
The dynamical behavior of fluxons propagating in the presence of an applied magnetic field on an overlap-geometry Josephson tunnel junction of length $5 \lambda_{J}$ having a McCumber $\beta_{c}=5 \pi$ is studied by numerical integration of the circuit equations of a 50 -section lumped RSJ-type (resistive shunted junction) model. Resonant propagating configurations corresponding to the first and third Fiske steps are found. The fundamental frequencies and power levels of the radiation emitted from one end when the junction is biased on the first and third Fiske steps and on the first zero-field step are comparable, but a magnetic field renders the power spectra at the two ends of the junction different.
\end{abstract}

\section{INTRODUCTION}

The zero-field steps, or dc current singularities, that appear (also) in the absence of applied magnetic fields in the dc current-voltage characteristics of Josephson tunnel junctions which are long in one dimension with respect to the Josephson penetration length $\lambda_{J}$ have by now been convincingly shown to be associated with the resonant propagation of fluxons in the junction, ${ }^{1-3}$ according to the mechanism first proposed by Fulton and Dynes. ${ }^{4}$ In this picture the first zero-field step (ZFS), which has a voltage asymptote of $\Phi_{0} \bar{c} / l$, where $\Phi_{0}$ is the magnetic flux quantum, $\bar{c}$ is the electromagnetic wave velocity within the junction, and $l$ is the length of the junction, is due to the propagation back and forth along the junction of a single fluxon; the second ZFS, whose voltage asymptote is twice that of the first, is due to two fluxons, etc.

In addition to the ZFS, when a dc magnetic field is applied in the plane of a Josephson tunnel junction (either long or short), a second set of current steps, called Fiske steps (FS), is observed in the current-voltage characteristic. Successive FS occur with a voltage-asymptote spacing just half that of the ZFS. For short junctions, the theory of FS developed by Kulik ${ }^{5}$ quite satisfactorily accounts for experimental observations; however, this is no longer the case for long junctions. ${ }^{6}$ Extensions of Kulik's theory to long junctions, framed in terms of cavity-mode interactions, have been formulated by various authors. ${ }^{7-10}$ These analyses are intended to be applicable to both FS and ZFS, and they have found varying degrees of success in explaining experimental observations.

The idea of applying the fluxon propagation model to the explanation of FS in long junctions was first suggested, but rejected as not physically feasible, by Fulton and Dunkleberger. ${ }^{11}$ It was reproposed, with an argument for its feasibility, by Samuelsen. ${ }^{12}$ The essential ingredients of this picture are the observation that an applied magnetic field renders the junction dynamical equation asymmetric through the boundary conditions, ${ }^{13}$ thus rendering wave propagation along the junction asymmetric, and the observation that the average junction voltage in a fluxon propagation mode depends only upon the time-averaged number of fluxons present, so that, for example, the first FS is consistent with a situation in which a single fluxon is present for half the time. Later, a numerical simulation result which supported Samuelsen's hypothesis was reported by Dueholm et al. ${ }^{14}$

The purpose of this paper is to contribute to a clarification of the situation through a numerical simulation of a long Josephson junction in a magnetic field. The results that emerge give further support to the Samuelsen mechanism. Specific predictions of the frequencies and power levels of the microwave radiation emitted by a junction currentbiased on a FS, as compared with the same quantities with the junction biased on a ZFS, are consistent with experimental measurements. Moreover, the results suggest further experimental measurements to check the proposed mechanism. 


\section{JUNCTION MODEL AND COMPUTATION TECHNIQUES}

The junction model and computational procedures employed in this study are essentially those used in a previous study of ZFS. ${ }^{1}$ An electrical representation of the model is shown in Fig. 1. The spatial dependence of the problem is taken into account by considering a 50-section lumped-circuit approximation to the junction. A junction length of $5 \lambda_{J}$ is assumed throughout this study. Dissipation is assumed to be due only to the linear resistor $R$ of Fig. 1. Accordingly, we assume simply that the powers radiated from the left and right ends of the junction at a given frequency are equal to the sum of the squares of the Fourier components $V_{\cos }^{2}+V_{\sin }^{2}$ at that frequency of the end-point voltages $V_{L}$ and $V_{R}$, respectively.

An overlap-geometry junction is assumed, implying that the bias current, $I_{B}$ per section, may be considered to be uniformly distributed along the junction. ${ }^{15}$ The effect of an externally applied dc magnetic field $B_{\text {ex }}$ in the plane of the junction and perpendicular to its long dimension is modeled by applying the dc current $I_{F}$ as shown in Fig. 1. The relation between the two quantities is $B_{\mathrm{ex}}=\mu_{0} I_{F} / w$, where $\mu_{0}$ is the permeability of free space and $w$ is the width of the junction in the field direction.

The Josephson element $J$ in Fig. 1 is characterized by the adiabatic Josephson equations

$$
\begin{aligned}
& I=I_{0} \sin \phi, \\
& \frac{d \phi}{d t}=\frac{2 \pi}{\Phi_{0}} v .
\end{aligned}
$$

Combining Eqs. (1a) and (1b) with the circuit equations of Fig. 1 results in, for section 1,

$$
\frac{\tau_{c}}{\tau_{\Delta}} \frac{d V_{1}}{d \tau}=\gamma+M+\lambda_{J}^{2}\left(\phi_{2}-\phi_{1}\right)-\sin \phi_{1}-V_{1}
$$

for section $j(1<j<50)$,

$$
\begin{aligned}
\frac{\tau_{c}}{\tau_{\Delta}} \frac{d V_{j}}{d \tau}= & \gamma+\lambda_{J}^{2}\left(\phi_{j-1}-2 \phi_{j}+\phi_{j+1}\right) \\
& -\sin \phi_{j}-V_{j}
\end{aligned}
$$

for section 50 ,

$$
\frac{\tau_{c}}{\tau_{\Delta}} \frac{d V_{50}}{d \tau}=\gamma-M+\lambda_{J}^{2}\left(\phi_{49}-\phi_{50}\right)-\sin \phi_{50}-V_{50},
$$

and for all sections $(1 \leq j \leq 50)$,

$$
\frac{d \phi_{j}}{d \tau}=\frac{\pi}{2} V_{j}
$$

Here $V$ is voltage $v$ normalized to $I_{0} R, \tau$ is time normalized to $\tau_{\Delta} \equiv \Phi_{0} / 4 I_{0} R$ (the inverse of the gap-sum frequency), $\tau_{c} \equiv R C, \quad \gamma \equiv I_{B} / I_{0}, \quad M \equiv I_{F} / I_{0}, \quad$ and $\lambda_{J}^{2} \equiv \Phi_{0} / 2 \pi L I_{0}$. The value of the parameter $\tau_{c} / \tau_{\Delta}$, which is a measure of the dissipation, is assumed to be 10 throughout this study; the value of the parameter $M$, which is a measure of the applied magnetic field, is fixed at 8.

Equations (2a)-(2d) correspond to a discretized version of the perturbed sine-Gordon equation, with boundary conditions given by $\phi_{x}(0, t)=\phi_{x}(l, t) \propto B_{\text {ex }}$, as employed by many other authors (see, e.g., Ref. 13). The time normalization employed in Eqs. (2a)-(2d) is the same as in Ref. 1, but it differs from that used by other authors, who measure time in units of the inverse plasma frequency $1 / \omega_{J} \equiv\left(C \Phi_{0} / 2 \pi I_{0}\right)^{1 / 2}$. It is trivial to show that the relation between the two time scales is given by $\omega_{J} \tau_{\Delta}=\left(2 \tau_{c} / \pi \tau_{\Delta}\right)^{-1 / 2}$. It is likewise trivial to show that the dissipative parameter $\tau_{c} / \tau_{\Delta}=2 \beta_{c} / \pi$, where $\beta_{c}$ is the usual McCumber parameter.

Equations (2a)-(2d) were integrated by means of a fourth-order Runge-Kutta routine using a fixed time increment of $0.01 \tau_{\Delta}$. The problem was integrated as an initial-value problem, and two types of initial conditions were employed:

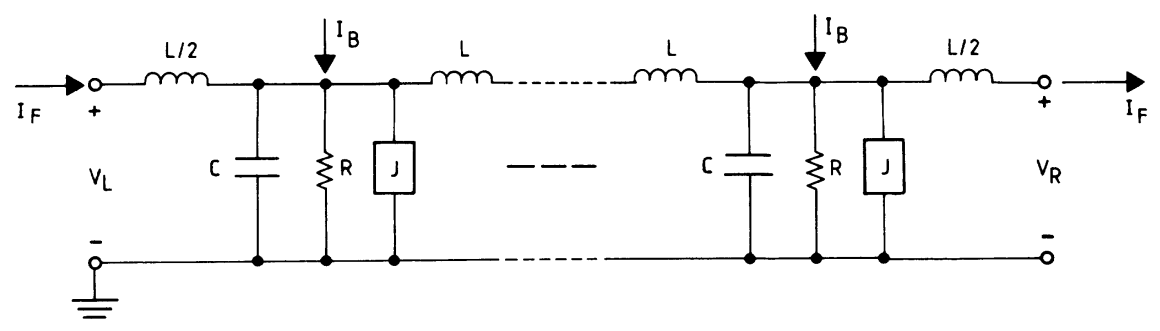

FIG. 1. Lumped circuit junction model. 

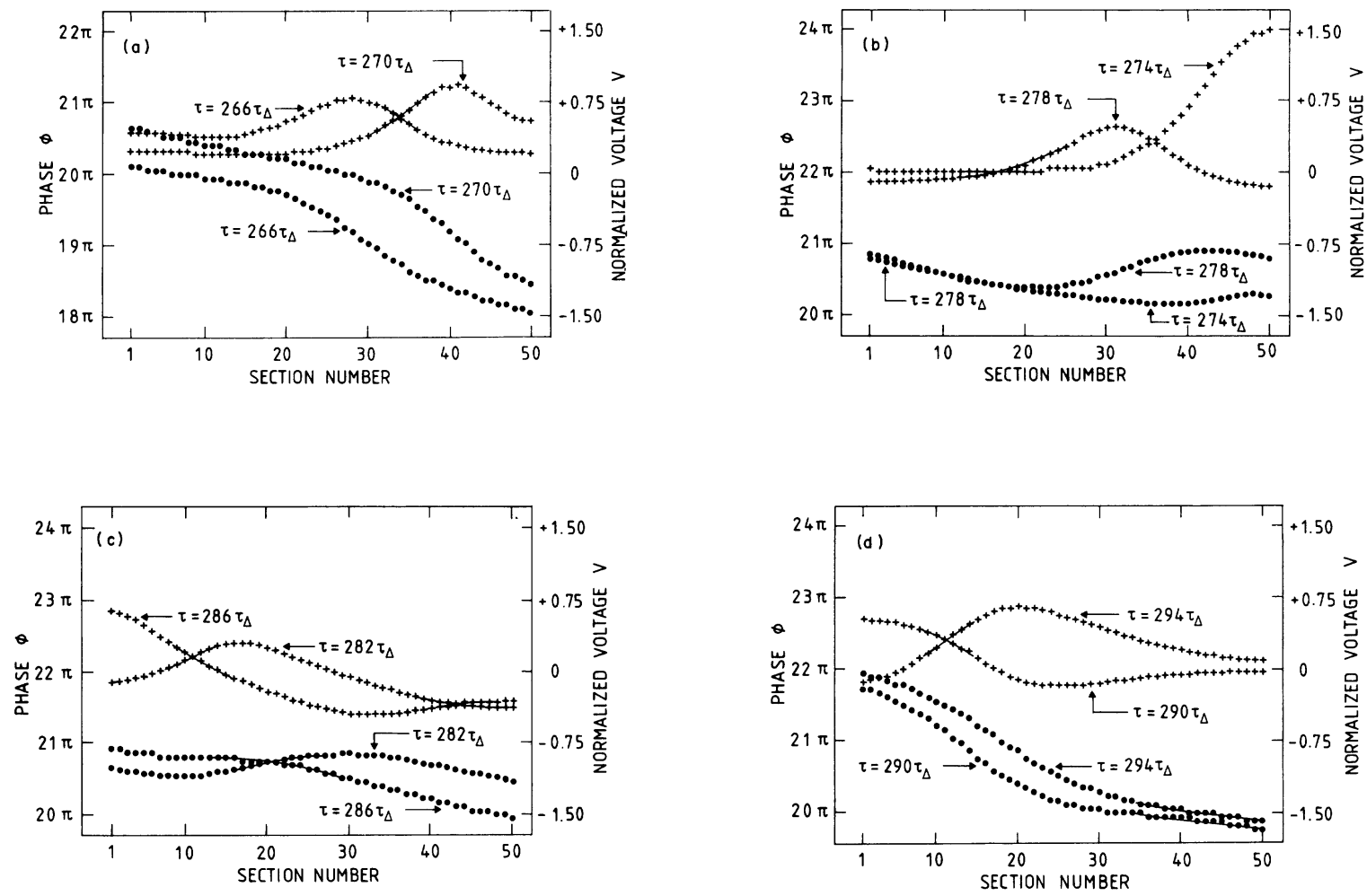

FIG. 2. Time evolution of FS-1 fluxon propagation for $\gamma=0.53$ and $M=8$. Circles: phase $\phi$; crosses: normalized voltage $V$. (a) $\tau=266 \tau_{\Delta}$ and $270 \tau_{\Delta}$; (b) $\tau=274 \tau_{\Delta}$ and $278 \tau_{\Delta}$; (c) $\tau=282 \tau_{\Delta}$ and $286 \tau_{\Delta}$ (d) $\tau=290 \tau_{\Delta}$ and $294 \tau_{\Delta}$. Apparent discontinuities are due to printer discretization.

(1) When searching for a first point on a given FS, the simple phase and voltage distributions shown in Fig. 2(a) of Ref. 1 were used. These distributions represent only a rough approximation to a single propagating fluxon, and their use constitutes a weakness in the calculations, as will be discussed below. (2) When searching for the upper (lower) extremity (in bias current) of a given FS, $\gamma$ was increased (decreased) by a small increment and the initial phase and voltage distributions were taken as the final distributions corresponding to the previous bias value. The integration was continued until the oscillation settled into a steady state, defined operationally as in Ref. 1. Normally, this required integrating to about $300 \tau_{\Delta}$ (about 9-10 complete oscillation periods).

After stopping the integration, the final two oscillation periods of the voltages at the two ends of the junction, $V_{L}$ and $V_{R}$, were recorded on a grid of approximately 500 equally spaced points. The average values of these voltages, $\left\langle V_{L}\right\rangle$ and $\left\langle V_{R}\right\rangle$, were determined using the fact that the average voltage over a period is proportional to the phase difference over that period, through the Josephson frequency relation, Eq. (2d). Physically, $\langle V\rangle$ must be constant along the entire junction; in practice, $\left\langle V_{L}\right\rangle$ and $\left\langle V_{R}\right\rangle$ were always equal to within better than $0.5 \%$.

The Fourier sine and cosine coefficients of $V_{L}$ and $V_{R}$ were then obtained by trapezoidal rule integration of the basic definitions of these coefficients over the grid. Since the integration was performed over two oscillation periods, the first evenorder harmonic was, in fact, the fundamental, and the absence of odd-order harmonics served as a further check that the oscillation had indeed reached a steady state.

\section{FLUXON OSCILLATIONS AND FISKE STEPS}

Figures 2 and 3 show a series of snapshots of the phase and voltage distributions along the junction over a single period of oscillation for two different modes of propagation. In Fig. 2(a), a fluxon is located near the center of the junction at $\tau=266 \tau_{\Delta}$ and is propagating toward the right (this is an antifluxon by the definition used in Ref. 1, but the dis- 

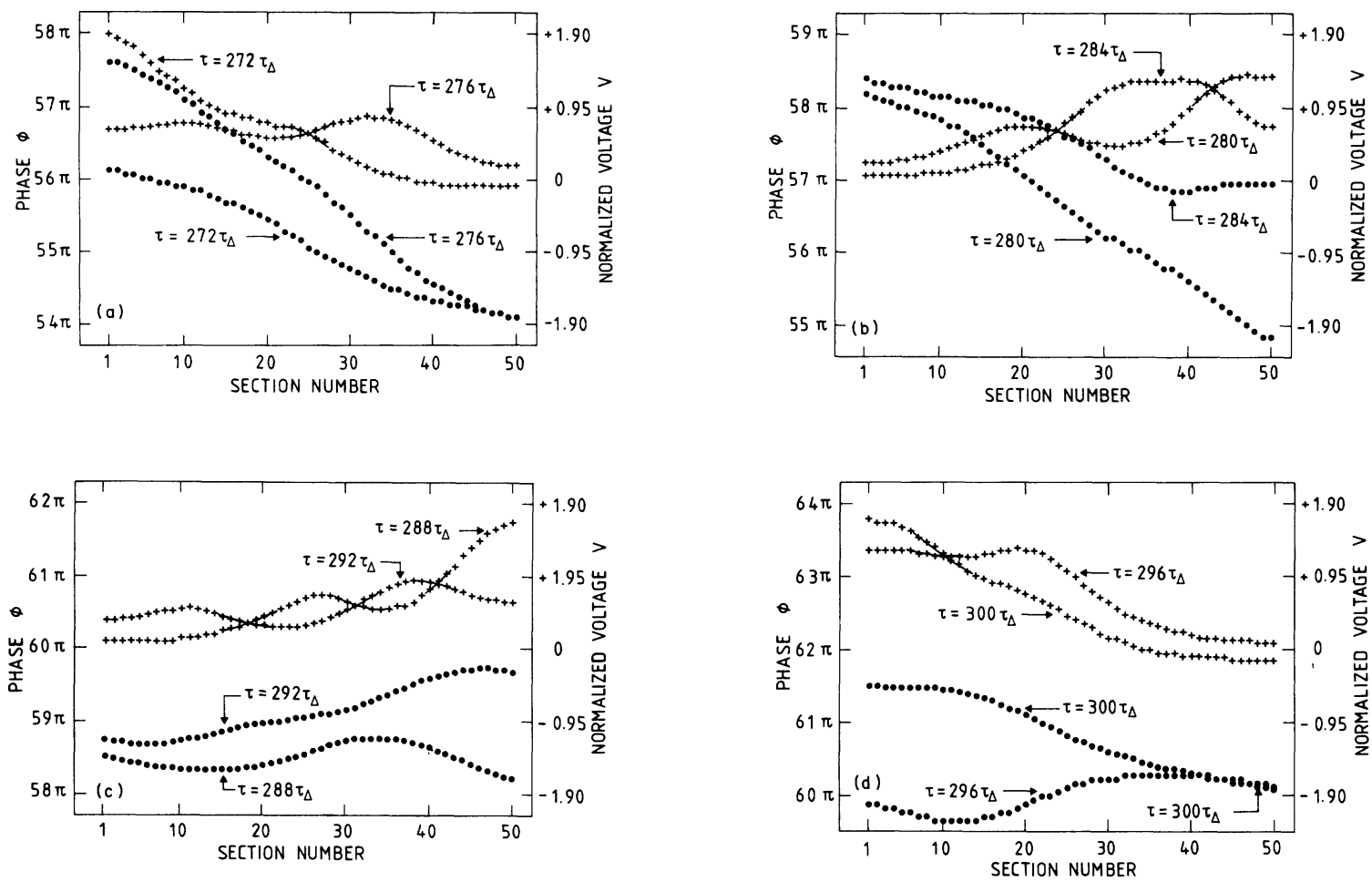

FIG. 3. Time evolution of FS-3 fluxon propagation for $\gamma=0.60$ and $M=8$. Circles: phase $\phi$; crosses: normalized voltage $V$. (a) $\tau=272 \tau_{\Delta}$ and $276 \tau_{\Delta}$; (b) $\tau=280 \tau_{\Delta}$ and $284 \tau_{\Delta}$; (c) $\tau=288 \tau_{\Delta}$ and $292 \tau_{\Delta}$; (d) $\tau=296 \tau_{\Delta}$ and $300 \tau_{\Delta}$. Apparent discontinuities are due to printer discretization.

tinction is arbitrary). Note that there is a net phase difference of $2 \pi$ along the junction and that the voltage is a well-defined pulse which is everywhere positive. At $\tau=270 \tau_{\Delta}$, the fluxon is approaching a reflection at the right end of the junction. In Fig. 2(b), this reflection has just taken place at $\tau=274 \tau_{\Delta}$, after which a clearly defined voltage pulse begins propagating to the left. Note, however, that the voltage waveform now goes both positive and negative, and that the net end-to-end phase difference is essentially zero. These facts suggest that the entity in question is a localized plasmon, or perhaps a plasmonbreather oscillation. ${ }^{16}$ In Fig. 2(c) this entity continues propagating to the left, and at $\tau=286 \tau_{\Delta}$ it is approaching a reflection at the left end. In Fig. 2(d) this reflection has just occurred at $\tau=290 \tau_{\Delta}$, whereupon propagation resumes toward the right. During the reflection, however, the net end-to-end phase difference has increased again toward $2 \pi$, so that at $\tau=294 \tau_{\Delta}$ the oscillation has completed almost one full cycle. As is evident from Fig. 2, the phase at any point along the junction advances by a total of $2 \pi$ during one oscillation period, as compared with the value of $4 \pi$ for the oscillation associ- ated with the first ZFS (cf. Fig. 2 of Ref. 1).

In Fig. 3 the situation is somewhat less "binary" inasmuch as the various propagating entities overlap one another, but the overall picture is still sufficiently clear. In Fig. 3(a), at $\tau=272 \tau_{\Delta}$ there is a net phase difference of $2 \pi$ along the junction and a voltage peak near section 21, corresponding to a fluxon moving to the right, but a second fluxon is rapidly entering from the left end. At $\tau=276 \tau_{\Delta}$ two voltage pulses are clearly visible, and the net end-to-end phase difference is about $3.5 \pi$, corresponding to something less than two fluxons in the junction. In Fig. 3(b) this packet has moved to the right at $\tau=280 \tau_{\Delta}$, with the first pulse approaching a reflection at the right end of the junction and the second one near section 21. During this portion of the oscillation the situation suggests, approximately, the propagation of two fluxons in a "bunched" configuration. ${ }^{17,18}$ At $\tau=284 \tau_{\Delta}$ the leading voltage pulse has just emerged from the reflection and is located near section 40, moving to the left; whereas the trailing pulse is located near section 33 and moving to the right. In Fig. 3(c) the two voltage pulses have exchanged positions at $\tau=288 \tau_{\Delta}$, with the leading 


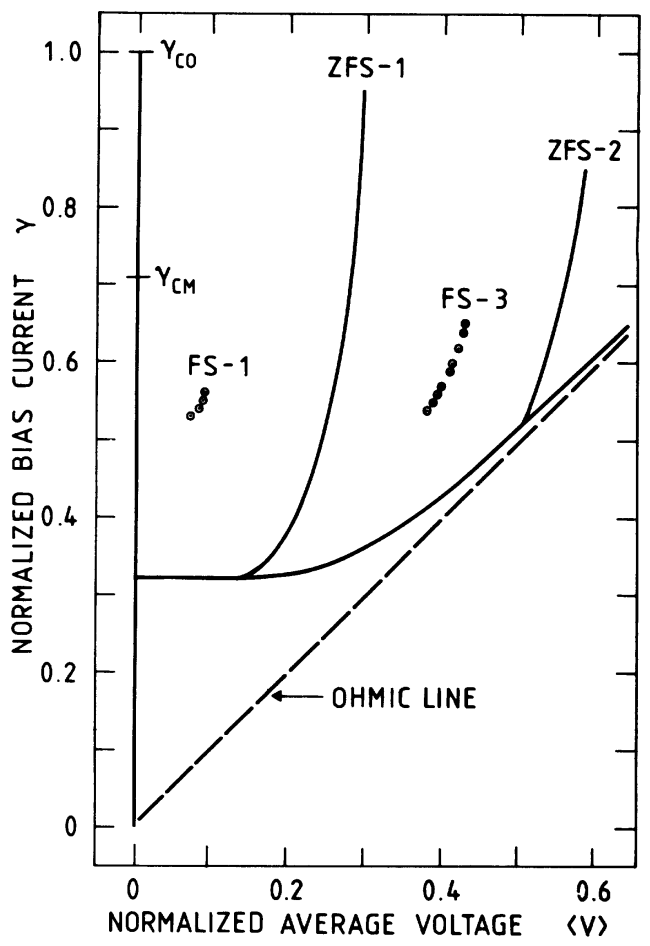

FIG. 4. Normalized current-voltage characteristic of the junction. Solid curve: magnetic field equal to zero, showing first two zero-field steps; circles: first and third Fiske steps for $M=8$. $\gamma_{\mathrm{CM}}$ is the field-reduced critical current value.

pulse now located near section 27 , moving to the left, and the trailing pulse just undergoing a reflection from the right end. Note that the net phase difference along the junction is at this point essentially zero. At $\tau=292 \tau_{\Delta}$ the two voltage pulses continue propagating to the left, and the net end-to-end phase difference has become approximately $\pi$. Interpretation of this situation is not completely unambiguous, but the propagation of a fluxonplasmon, fluxon-breather, or fluxon-plasmonbreather combination is suggested. In Fig. 3(d) the

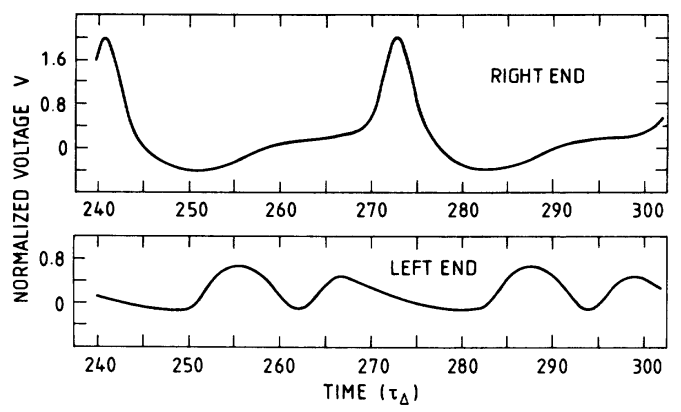

FIG. 5. End-point voltage waveforms of FS-1 fluxon oscillation for $\gamma=0.53$ and $M=8$.

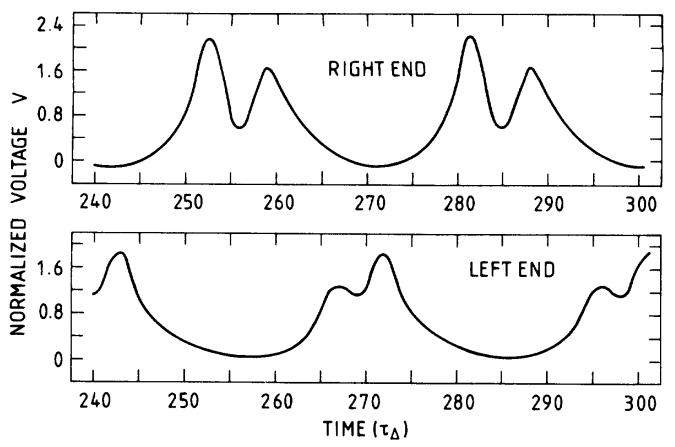

FIG. 6. End-point voltage waveforms of FS-3 fluxon oscillation for $\gamma=0.60$ and $M=8$.

leading voltage pulse is just undergoing a reflection from the left end of the junction at $\tau=296 \tau_{\Delta}$, and the trailing pulse is located near section 19 and still moving to the left. At $\tau=300 \tau_{\Delta}$ the leading pulse, barely distinguishable, is located near section 17 , moving again toward the right, whereas the trailing pulse is approaching a reflection at the left end. The net end-to-end phase difference is now approximately $1.5 \pi$, and the oscillation has completed almost one full cycle. Figure 3 shows clearly that during one full period of this oscillation the phase at any point along the junction advances by a total of $6 \pi$.

Figures 2 and 3 show each of the two modes of propagation for a single value of the bias current. Each of these two modes, however, exists over a certain range of $\gamma$. This fact is displayed in Fig. 4, which shows the two loci of points, indicated as circles, labeled, respectively, as FS-1 and FS-3 in the $\gamma-\langle V\rangle$ plane. The solid curve, for reference, is the current-voltage characteristic of the junction in the absence of magnetic field, taken from Ref. 1. The positions of the two groups of circles relative to the first two zero-field steps, ZFS-1 and ZFS-2, confirm that FS-1 and FS-3 are, respectively, the first and third Fiske steps. The point $\gamma_{\mathrm{CM}}$ at $\langle V\rangle=0$, incidentally, is the value to which the critical current has been reduced by the magnetic field $M=8$.

At this point we note that there should also exist a second FS corresponding approximately in voltage position to ZFS-1, but we have not been able to find this FS numerically. We believe that the reason for this is that the initial condition employed is not really appropriate to finding FS-2. We have observed in calculating FS-1 and FS-3 that the outcome of the simulation depends, at least sometimes, quite strongly on the initial condition employed: If the initial condition is "too far" (by some measure) from the final propagating configuration, the junction tends to switch to some other mode of propagation. 

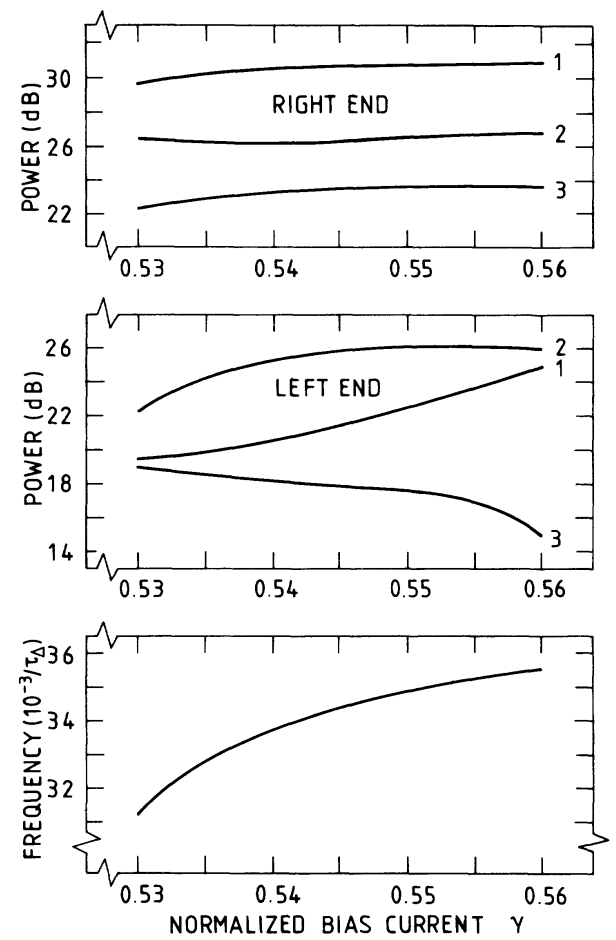

FIG. 7. Power spectra (first three harmonics) of the radiation emitted from the junction ends, normalized to $V_{L}, V_{R}=0.04 / \pi$. The frequency indicated is that of the fundamental component. The junction is biased on the first Fiske step.

\section{POWER SPECTRA}

Figures 2 and 3 clearly show that the two modes of propagation associated with FS-1 and FS-3 are asymmetric. This fact is underlined in Figs. 5 and 6 , which show the temporal evolution of the two end-point voltages $V_{L}$ and $V_{R}$ in the two cases corresponding to Figs. 2 and 3, respectively.

Since we assume that the power radiated from an end of the junction is proportional to the square of the voltage at that end, it is clear that Figs. 5 and 6 imply that the spectra of the radiation emitted from the two ends are quite different. That this is indeed the case is indicated in Figs. 7 and 8, which show the power spectra, calculated as described above, for the first three Fourier components of the voltages $V_{L}$ and $V_{R}$, corresponding, respectively, to FS-1 and FS-3 as a function of the bias current. The frequency shown in the lower part of the figures is that of the fundamental (labeled 1) Fourier component in each case. The power levels have been normalized to an arbitrary value of $V_{L}, V_{R}=0.04 / \pi$.

The main conclusions to be drawn from Figs. 7
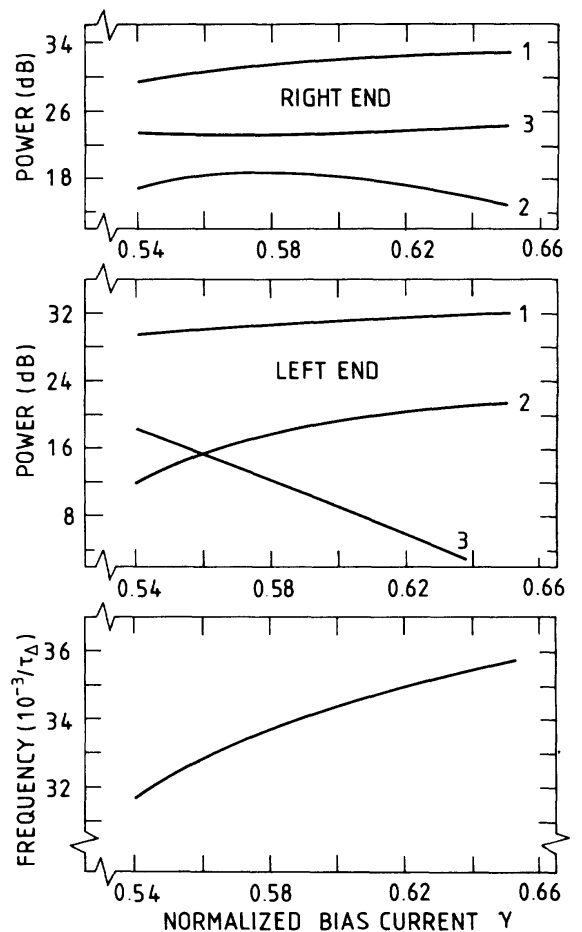

FIG. 8. Power spectra (first three harmonics) of the radiation emitted from the junction ends, normalized to $V_{L}, V_{R}=0.04 / \pi$. The frequency indicated is that of the fundamental component. The junction is biased on the third Fiske step.

and 8 are the following: (1) The fundamental frequencies associated with FS-1 and FS-3 are the same; moreover, they are the same as that associated with ZFS-1 [cf. Fig. 5(a) of Ref. 1]. This fact has already been confirmed by experimental observations. ${ }^{14}$ (2) The power levels of the radiation emitted by a junction biased on a FS are comparable, at least in the field-aided direction, with those obtained with the junction biased on a ZFS (cf. Fig. 5 of Ref. 1). This fact is also consistent with experimental observations. ${ }^{14}$ (3) The asymmetries in the power spectra of the radiation emitted from the two ends of the junction may well be large enough to be measurable. One way of doing this would be to couple to a single end of the junction and measure the radiation with both polarities of the magnetic field.

\section{COMMENTS}

The present work is intended as a contribution to the understanding of the dynamics of long Josephson tunnel junctions. We believe that fluxon propa- 
gation is the basic physical mechanism underlying all of the various phenomena observed in connection with such long junctions, and that cavity-mode interaction analyses are essentially mathematical approximations that, as they become more refined, tend toward the same picture, being particularly suitable in the limit of short junctions. We propose that a detailed comparison of the results of experimental measurements on real junctions with those from careful numerical simulations, similar to those recently reported by Lomdahl et al. ${ }^{3}$ in connection with ZFS, should be an important objective for future studies.

\section{ACKNOWLEDGMENTS}

We are grateful to J. Mygind, M. R. Samuelsen, and A. C. Scott for helpful and stimulating discussions. One of us (A.F.) acknowledges financial support from the Deutscher Akademischer Austauschdienst. Another of us (R.D.P.) was supported in part by the Consiglio Nazionale delle Ricerche through the Gruppo Nazionale di Struttura della Materia and the Progetto Finalizzato "Superconduttività," and in part by the European Research Office of the United States Army through Contract No. DAJA37-82-C-0057.
"Permanent address: Istituto di Fisica, Università degli Studi di Salerno, I-84100 Salerno, Italy.

1S. N. Erné and R. D. Parmentier, J. Appl. Phys. 51, 5025 (1980).

${ }^{2}$ B. Dueholm, O. A. Levring, J. Mygind, N. F. Pedersen, O. H. Soerensen, and M. Cirillo, Phys. Rev. Lett. 46, 1299 (1981).

${ }^{3}$ P. S. Lomdahl, O. H. Soerensen, and P. L. Christiansen, Phys. Rev. B 25, 5737 (1982).

${ }^{4}$ T. A. Fulton and R. C. Dynes, Solid State Commun. 12, 57 (1973).

5I. O. Kulik, Zh. Tekh. Fiz. 37, 157 (1967) [Sov. Phys.Tech. Phys. 12, 111 (1967)].

${ }^{6} \mathrm{G}$. Paternò and J. Nordman, J. Appl. Phys. 49, 2456 (1978).

${ }^{7}$ K. Takanaka, Solid State Commun. 29, 443 (1979).

${ }^{8}$ Y.-S. Gou and C.-S. Chung, J. Low Temp. Phys. 37, 367 (1979).

${ }^{9}$ K. Enpuku, K. Yoshida, and F. Irie, J. Appl. Phys. 느, 344 (1981).
10J.-J. Chang, J. T. Chen, and M. R. Scheuermann, Phys. Rev. B $\underline{25}, 151$ (1982).

${ }^{11}$ T. A. Fulton and L. N. Dunkleberger, Rev. Phys. Appl. 9, 299 (1974)

${ }^{12} \mathrm{M}$. R. Samuelsen (private communication).

${ }^{13}$ O. H. Olsen and M. R. Samuelsen, J. Appl. Phys. 2, 6247 (1981).

${ }^{14}$ B. Dueholm, E. Joergensen, O. A. Levring, J. Mygind, N. F. Pedersen, M. R. Samuelsen, O. H. Olsen, and M. Cirillo, Physica 108B, 1303 (1981).

${ }^{15}$ A. Barone, W. J. Johnson, and R. Vaglio, J. Appl. Phys. 46, 3628 (1975).

${ }^{16}$ R. D. Parmentier, in Solitons in Action, edited by K. Lonngren and A. Scott (Academic, New York, 1978), pp. 173-199.

${ }^{17}$ S. N. Erné and R. D. Parmentier, J. Appl. Phys. 52, 1091 (1981).

${ }^{18}$ P. L. Christiansen, P. S. Lomdahl, A. C. Scott, O. H. Soerensen, and J. C. Eilbeck, Appl. Phys. Lett. 9, 108 (1981). 